\title{
Supraferromagnetic correlations in clusters of magnetic nanoflowers
}

Cite as: Appl. Phys. Lett. 115, 132406 (2019); https://doi.org/10.1063/1.5121234

Submitted: 24 July 2019 . Accepted: 12 September 2019 . Published Online: 25 September 2019

P. Bender (iD), D. Honecker (iD), and L. Fernández Barquín (D)
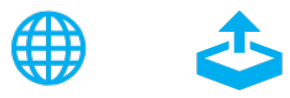

Export Citation

CrossMark

\section{ARTICLES YOU MAY BE INTERESTED IN}

Magnetic field orientation dependent dynamic susceptibility and Brownian relaxation time of magnetic nanoparticles

Applied Physics Letters 115, 133102 (2019); https://doi.org/10.1063/1.5120609

Magnon drag effect in Fe-Co alloys

Journal of Applied Physics 126, 125107 (2019); https://doi.org/10.1063/1.5117165

Temperature dependent magnetoelectric (ME) response in press-fit FeNi/PZT/Ni self-biased ring composite

Journal of Applied Physics 126, 094102 (2019); https://doi.org/10.1063/1.5108708

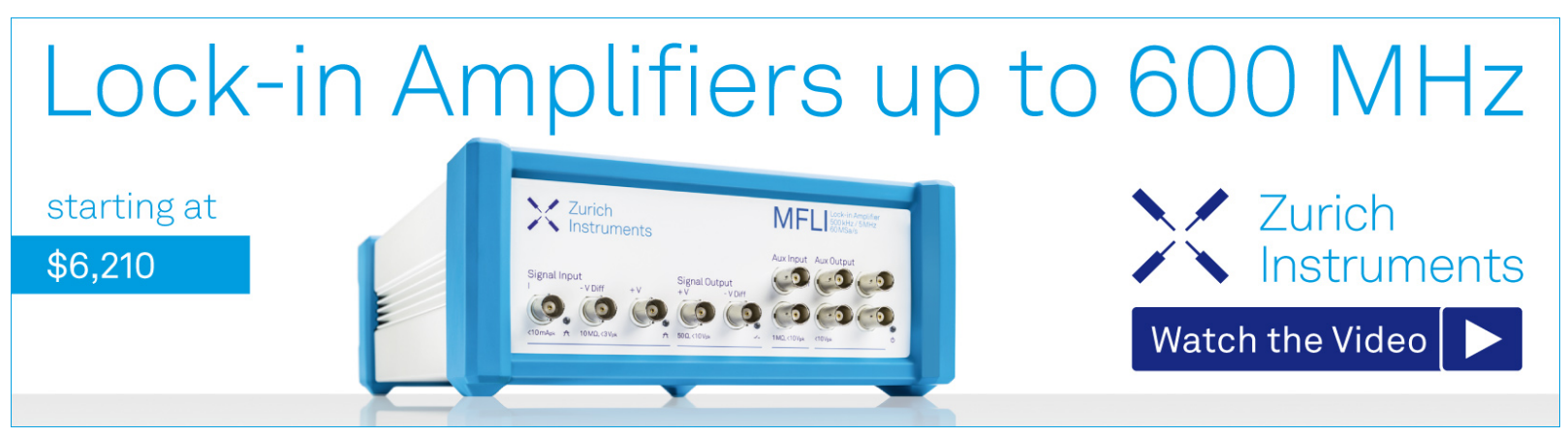




\title{
Supraferromagnetic correlations in clusters of magnetic nanoflowers
}

\author{
Cite as: Appl. Phys. Lett. 115, 132406 (2019); doi: 10.1063/1.5121234 \\ Submitted: 24 July 2019 . Accepted: 12 September 2019 . \\ Published Online: 25 September 2019
}

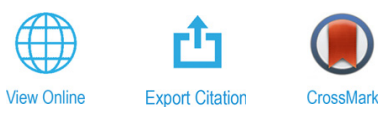

\author{
P. Bender, ${ }^{1, a)}$ (D) D. Honecker, ${ }^{2, b)}$ (D) and L. Fernández Barquín ${ }^{3}$ (D)
}

\author{
AFFILIATIONS \\ ${ }^{7}$ Physics and Materials Science Research Unit, University of Luxembourg, L-1511 Luxembourg, Grand Duchy of Luxembourg \\ ${ }^{2}$ Institut Laue-Langevin, 38042 Grenoble, France \\ ${ }^{3}$ Departamento CITIMAC, Faculty of Science, University of Cantabria, 39005 Santander, Spain

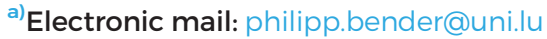

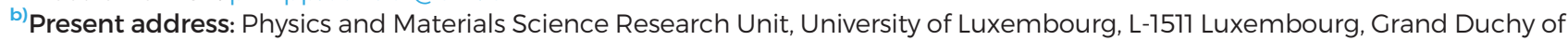 \\ Luxembourg.
}

\begin{abstract}
Magnetic nanoflowers are densely packed aggregates of superferromagnetically coupled iron oxide nanocrystallites, which excel during magnetic hyperthermia experiments. Here, we investigate the nature of the moment coupling within a powder of such nanoflowers using spin-resolved small-angle neutron scattering. Within the powder, the nanoparticles are agglomerated to clusters, and we can show that the moments of neighboring nanoflowers tend to align parallel to each other. Thus, the whole system resembles a hierarchical magnetic nanostructure consisting of three distinct levels, i.e., (i) the ferrimagnetic nanocrystallites as building blocks, (ii) the superferromagnetic nanoflowers, and (iii) the supraferromagnetic clusters of nanoflowers. We surmise that such a supraferromagnetic coupling explains the enhanced magnetic hyperthermia performance in the case of interacting nanoflowers.
\end{abstract}

Published under license by AIP Publishing. https://doi.org/10.1063/1.5121234

The working principle of magnetic hyperthermia (MHT) is to administer a moderate quantity of magnetic nanoparticles within tumors and to heat them up by applying alternating magnetic fields with clinically acceptable parameters [i.e., comparatively high frequencies $\gtrsim 100 \mathrm{kHz}$ but low amplitudes $\lesssim 20 \mathrm{mT}$ (Refs. 1 and 2)] to kill the tumors. Additionally, a magnetomechanical actuation of the embedded particles may disrupt the cytoskeleton and lead to cell death. ${ }^{3,4}$ In physiological environments, nanoparticles usually agglomerate, which can significantly modify their magnetic properties compared to the dilute noninteracting case ${ }^{5,6}$ and which in turn may alter their heating behavior. ${ }^{7-10}$ Depending on the characteristics of the individual particles and the field parameters, such a clustering can either improve or impair the MHT performance. ${ }^{11-15}$ In fact, it was observed for the so-called nanoflowers, which are densely packed aggregates of iron oxide crystallites, that they excel during MHT experiments compared to the single-crystals ${ }^{16}$ and other systems such as magnetosomes. ${ }^{17}$ This intriguing result motivated numerous studies regarding synthesis and characterization of such flower-shaped particles. ${ }^{18-23}$ It can be shown that an exchange coupling between the cores leads to a superferromagnetic magnetization state ${ }^{24}$ within the individual nanoflowers, ${ }^{25}$ but with a significant internal spin disorder caused by the high defect density, e.g., due to the grain boundaries. ${ }^{26}$ It is speculated that such a disordered state enables an increased excitation of the moments, ${ }^{27,28}$ similar to other defect-rich particles. ${ }^{29}$ When introduced into tumors, it is safe to assume that the nanoflowers will agglomerate to clusters, and thus interparticle interactions will be relevant. ${ }^{30}$ In Bender et al., ${ }^{31}$ we could show for homogeneous superparamagnetic nanoparticles a predominance for antiferromagnetic-like moment correlations within particle clusters via polarized small-angle neutron scattering (SANS). In this work, we use the same approach to determine the nature of the moment coupling within a powder of iron oxide nanoflowers.

The synomag-D nanoflowers were supplied by micromod Partikeltechnologie $\mathrm{GmbH}$. They consist predominately of $\gamma-\mathrm{Fe}_{2} \mathrm{O}_{3}$ and are coated with dextran. A detailed study of these particles can be found in Bender et al., ${ }^{27}$ which showed that they are around $39 \mathrm{~nm}$ in size and consist of crystallites with sizes ranging from 5 to $15 \mathrm{~nm}$. Transmission electron microscopy (TEM) images were taken with an FEI Titan 80-300 TEM, for which the sample was prepared by putting a small droplet of the dilute dispersion of the particles on a carbon-coated copper grid. Figure 1 shows a typical TEM image of the nanoflowers, in which they are agglomerated to small clusters of 3 and 9 particles, 


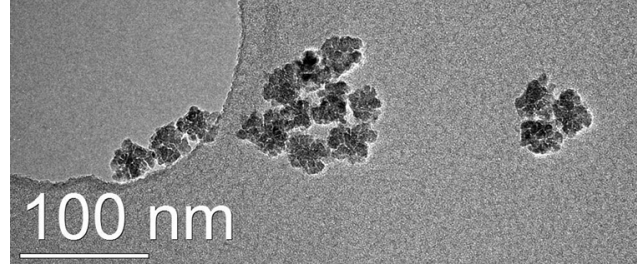

FIG. 1. TEM image of three separate clusters of nanoflowers. The nuclear SANS results indicate that within the particle powder large clusters with sizes $>160 \mathrm{~nm}$ exist.

respectively. As can be seen, the nanoparticles are irregular in shape and around $30-40 \mathrm{~nm}$ in size.

The polarized SANS experiment of the nanoflower powder ${ }^{32}$ was performed with the instrument D33 at the Institut Laue-Langevin (ILL), Grenoble (France) ${ }^{33}$ at room-temperature using a mean wavelength of $\lambda=0.6 \mathrm{~nm}(\Delta \lambda / \lambda=10 \%)$ and a detector distance of $10.3 \mathrm{~m}$. We employed longitudinal neutron-spin analysis (POLARIS) to collect the four spin-resolved intensities $I^{++}(\mathbf{q}), I^{--}(\mathbf{q}), I^{+-}(\mathbf{q})$, and $I^{-+}(\mathbf{q})$, where + denotes the polarization state "spin-up." This approach enables the separation of nuclear and magnetic scattering contributions and was applied in several studies to investigate magnetic nanoparticle ensembles. ${ }^{31,34,35}$ A homogeneous magnetic field $\mathbf{H}$ was applied perpendicular to the neutron beam $(\mathbf{H} \perp \mathbf{k})$ with a field amplitude of $\mu_{0} H=2 \mathrm{mT}$, which was necessary to maintain the neutron beam polarization.

Figures 2(a) and 2(b) display the 2D scattering patterns of the non-spin-flip (nsf) cross section $I^{--}(\mathbf{q})$ and of the spin-flip (sf) cross section $I^{+-}(\mathbf{q})$, respectively. For the geometry $\mathbf{H} \perp \mathbf{k}$, the nsf cross sections can be written as

$$
\begin{aligned}
I^{ \pm \pm}(\mathbf{q}) \propto & |\tilde{N}|^{2}+b_{\mathrm{h}}^{2}\left|\tilde{M}_{z}\right|^{2} \sin ^{4} \Theta+b_{\mathrm{h}}^{2}\left|\tilde{M}_{y}\right|^{2} \sin ^{2} \Theta \cos ^{2} \Theta \\
& -b_{\mathrm{h}}^{2}\left(\tilde{M}_{y} \tilde{M}_{z}^{*}+\tilde{M}_{z} \tilde{M}_{y}^{*}\right) \sin ^{3} \Theta \cos \Theta \\
& \mp b_{\mathrm{h}}\left(\tilde{N} \tilde{M}_{z}^{*}+\tilde{N}^{*} \tilde{M}_{z}\right) \sin ^{2} \Theta \\
& \pm b_{\mathrm{h}}\left(\tilde{N} \tilde{M}_{y}^{*}+\tilde{N}^{*} \tilde{M}_{y}\right) \sin \Theta \cos \Theta,
\end{aligned}
$$

where $\Theta$ is the angle between the scattering vector $\mathbf{q}=\left(0, q_{y}, q_{z}\right)$ and the magnetic field $\mathbf{H}$ and $b_{\mathrm{h}}=2.7 \times 10^{-15} \mathrm{~m} / \mu_{\mathrm{B}}$, with $\mu_{\mathrm{B}}$ being the Bohr magneton. Hence, in Figs. 2(a) and 2(b), the field was applied along $\Theta=0^{\circ}$. Moreover, $\tilde{N}(\mathbf{q})$ and $\tilde{\mathbf{M}}=\left[\tilde{M}_{x}(\mathbf{q}), \tilde{M}_{y}(\mathbf{q})\right.$, $\tilde{M}_{z}(\mathbf{q})$ ] are the Fourier transforms of the nuclear scattering length density and the magnetization vector field in the $x$-, $y$-, and $z$-directions, respectively, and the index ${ }^{*}$ denotes the complex conjugate. One remarkable advantage of POLARIS is that the purely nuclear scattering can be accessed without further assuming a saturated magnetic system (absence of misaligned moments). To be precise, the purely nuclear cross section $I^{\text {nuc }}(q) \propto|\tilde{N}|^{2}$ can be determined, in the case of isotropic structures, from the sector parallel to $\mathbf{H}$ of the nsf intensities. (a) nsf cross section - $^{--}$(q)
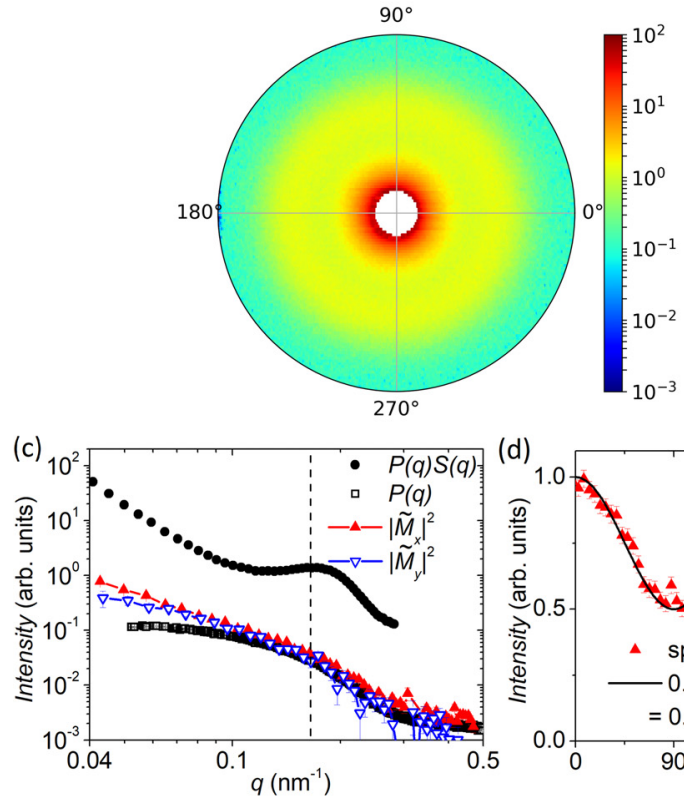

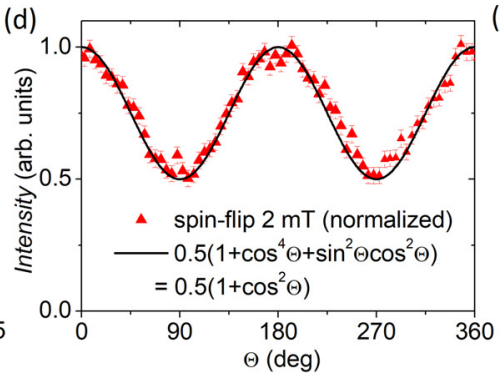

(b)
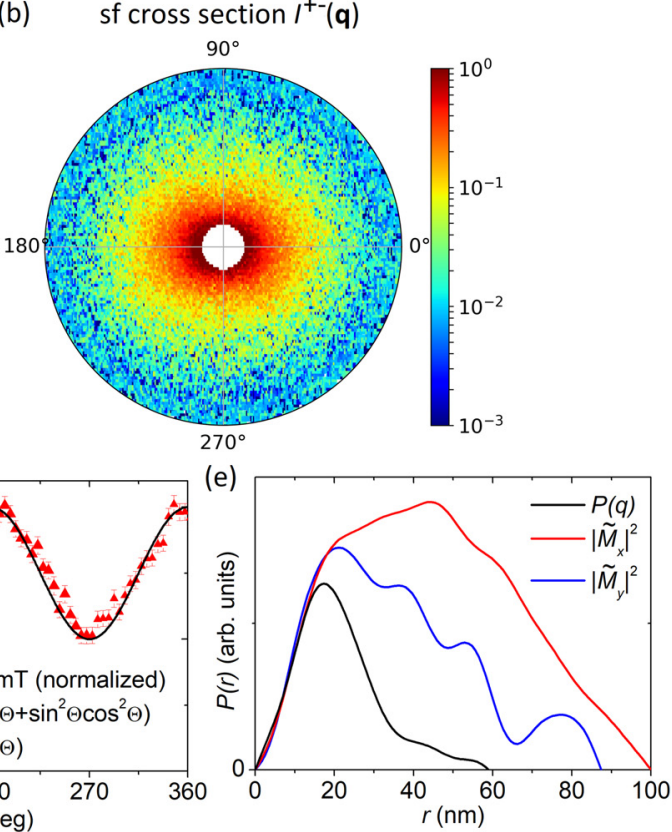

FIG. 2. Polarized SANS analysis of the nanoflower powder. The magnetic field with $\mu_{0} H=2 \mathrm{mT}$ was applied along $\Theta=0^{\circ}$, and the total accessible $q$-range was around $0.03-0.3 \mathrm{~nm}^{-1}$. (a) $2 \mathrm{D}$ scattering pattern of the nsf cross section $I^{--}$(q). (b) $2 \mathrm{D}$ scattering pattern of the sf cross section $l^{\prime f}(\mathbf{q})$. (c) Purely nuclear $1 \mathrm{D}$ cross section $l^{\text {nuc }}(q) \propto$ $P(q) S(q)$ extracted from $I^{--}(\mathbf{q})$ (sector parallel to $\mathrm{H}, \Theta=0^{\circ} \pm 10^{\circ}$ ) of the powder, the nuclear cross section $I^{\text {nuc }}(q) \propto P(q)$ determined from $I^{--}(\mathbf{q})$ of the dilute colloidal dispersion (from Bender et al. ${ }^{2}$ ) and the magnetic cross sections $\left|\tilde{M}_{x}\right|^{2}$ and $\left|\tilde{M}_{y}\right|^{2}$ extracted from the sf cross section $\mid \beta^{f}(\mathbf{q})$. The dashed line at $q=0.17 \mathrm{~nm}{ }^{-1}$ indicates the border between the intraparticle length scale (high $q$ ) and the interparticle length scale (low $q)$. (d) $\left.\right|^{s f}(\mathbf{q})$ integrated over the whole $q$-range as a function of $\Theta$. (e) The correlation functions $P(r)$ extracted by indirect Fourier transforms of the $1 \mathrm{D}$ nuclear scattering cross section $P(q)$ of the colloid (from Bender et al. ${ }^{27}$ ) and the magnetic cross sections $\left|\tilde{M}_{x}\right|^{2}$ and $\left|\tilde{M}_{y}\right|^{2}$. 
The sf intensities, on the other hand, are of purely magnetic origin. We assume for our sample that chiral scattering terms can be neglected, ${ }^{36}$ and thus we can write $I^{\mathrm{sf}}(\mathbf{q})=I^{+-}(\mathbf{q})=I^{-+}(\mathbf{q})$, with $(\text { for } \mathbf{H} \perp \mathbf{k})^{3}$

$$
\begin{aligned}
I^{\mathrm{sf}}(\mathbf{q}) \propto & \left|\tilde{M}_{x}\right|^{2}+\left|\tilde{M}_{y}\right|^{2} \cos ^{4} \Theta+\left|\tilde{M}_{z}\right|^{2} \sin ^{2} \Theta \cos ^{2} \Theta \\
& -\left(\tilde{M}_{y} \tilde{M}_{z}^{*}+\tilde{M}_{z} \tilde{M}_{y}^{*}\right) \sin \Theta \cos ^{3} \Theta .
\end{aligned}
$$

The nsf intensity in Fig. 2(a) exhibits basically no anisotropy, indicating the dominance of the isotropic nuclear scattering and thus verifying a randomly oriented microstructure. The purely nuclear $1 \mathrm{D}$ cross section $I^{\text {nuc }}(q)$ is determined from the sector parallel to magnetic field of $I^{--}(\mathbf{q})$ and is plotted in Fig. 2(c). As can be seen, $I^{\text {nuc }}(q)$ exhibits a peak at around $q=0.17 \mathrm{~nm}^{-1}$. For particle ensembles, the total nuclear cross section is usually written as $I^{\text {nuc }}(q) \propto P(q) S(q)$, where $P(q)$ is the particle form factor and $S(q)$ the structure factor arising from the particle arrangement. ${ }^{38}$ For comparison, in Fig. 2(c), we also plot the purely nuclear scattering cross section of the same nanoflowers in dilute colloidal dispersion from Bender et al. ${ }^{27}$ In this case, there is no significant structure formation, and thus $I^{\text {nuc }}(q) \propto P(q)$. The observed peak for $I^{\text {nuc }}(q)$ of the powder can be thus attributed to interparticle correlations, which implies an average center-to-center distance between the nanoflowers of $2 \pi / 0.17 \mathrm{~nm}^{-1}=36 \mathrm{~nm}$ (nearest neighbor correlations). ${ }^{39,40}$ This estimation is in good agreement with our previous analysis in Bender et al., ${ }^{27}$ where we determined an average particle size of around $39 \mathrm{~nm}$. For $q \rightarrow 0$ (i.e., the interparticle length scale), the forward scattering intensity increases, which indicates the presence of larger structures within the samples. ${ }^{39}$ Thus, we can conclude that no long-range order exists but that the nanoflowers within the powder are agglomerated to large clusters with average sizes outside the minimal $q$-resolution, i.e., average cluster sizes of $>160 \mathrm{~nm}$.

The sf intensity $I^{\text {sf }}(\mathbf{q})$ in Fig. 2(b) exhibits a well-pronounced anisotropy, and in Fig. 2(d), we plot $I^{\mathrm{sf}}(q)$ integrated over the whole $q$-range as a function of $\Theta$. The functional form is well described by the trigonometric terms from Eq. (2) without the linear term, which implies equal magnetization along the $x$-, $y$-, and $z$-directions and a zero net magnetization. This is expected because the sample was in the demagnetized state (i.e., the powder was not exposed to a magnetic field prior to the polarized SANS experiment) and $2 \mathrm{mT}$ is not sufficient to significantly align the moments (as a reminder, the low magnetic field had to be applied to remain the polarization of the neutron beam). In Fig. 2(c), we plot $I^{\text {sf }}(\mathbf{q})$ determined perpendicular to the field direction, i.e., $I^{\mathrm{sf}}\left(q, \Theta=90^{\circ}\right) \propto\left|\tilde{M}_{x}\right|^{2}$, and the difference between $I^{\text {sf }}\left(q, \Theta=90^{\circ}\right)$ and $I^{\text {sf }}\left(q, \Theta=0^{\circ}\right)$, i.e., $\left|\tilde{M}_{y}\right|^{2}$. Both cross sections are in the high $q$-range (i.e., the intraparticle $q$-range) basically identical to each other and to the nuclear particle form factor $P(q)$. This confirms the superferromagnetic magnetization state within the individual nanoflowers. In the interparticle $q$-range $\left(q<0.17 \mathrm{~nm}^{-1}\right)$, however, both $\left|\tilde{M}_{x}\right|^{2}$ and $\left|\tilde{M}_{y}\right|^{2}$ start to deviate from $P(q)$ and increase strongly with decreasing $q$. Additionally, it can be observed in Fig. 2(c) that in the low $q$-range, $\left|\tilde{M}_{x}\right|^{2}$ significantly deviates from $\left|\tilde{M}_{y}\right|^{2}$. This can be attributed to the anisotropy of the magnetic structure factor and indicates a disordered microstructure without a short range pseudocrystalline order. ${ }^{41}$ The deviation of both magnetic contributions $\left|\tilde{M}_{x}\right|^{2}$ and $\left|\tilde{M}_{y}\right|^{2}$ from $P(q)$ is evidence for interparticle moment correlations between neighboring nanoflowers. To reveal the nature of these interactions, we extracted the underlying magnetic correlation functions $P(r)$ from the scattering intensities by indirect Fourier transforms. ${ }^{42}$ As can be seen in Fig. 2(e), for the two magnetic contributions $\left|\tilde{M}_{x}\right|^{2}$ and $\left|\tilde{M}_{y}\right|^{2}$, we obtain positive values for $P(r)$ for length scales well above the nanoflower size $(r \geq 36 \mathrm{~nm})$, which indicates positive correlations between the moments of neighboring nanoflowers. This can be interpreted as evidence for a supraferromagnetic magnetization state within the clusters of these superferromagnetic nanoflowers.

To conclude, we performed a spin-resolved SANS study on a powder of iron oxide nanoflowers, which enables the separation of nuclear and magnetic scattering contributions. Analysis of the nuclear SANS data shows that the nanoflowers are agglomerated to large clusters. The magnetic scattering contributions then indicate that the moments between neighboring particles are preferentially aligned parallel to each other. We interpret this as evidence for a supraferromagnetic magnetization state within the clusters of nanoflowers. Considering that the nanoflowers itself are aggregates of superferromagnetically coupled crystallites, the whole system can be thus regarded as a hierarchical magnetic nanostructure consisting of three distinct levels, i.e., (i) the ferrimagnetic nanocrystallites as building blocks, (ii) the superferromagnetic nanoflowers, and (iii) the supraferromagnetic clusters of nanoflowers. It can be assumed that such supraferromagnetic correlations increase the low-field susceptibility of the ensemble and thus its MHT performance compared to the dilute, noninteracting ensemble. Indeed, we surmise that our observation explains the intriguing result in Sakellari et al. ${ }^{19}$ where for colloidal dispersions of 50-nm nanoflowers, an increased heating with the increasing particle concentration was detected, which is in contrast to other nanoparticle ensembles for which usually increasing interactions result in a decrease in the MHT performance. ${ }^{43,44}$ Considering that in physiological environments usually a clustering of immersed nanoparticles occurs, it is a promising result for such nanoflowers that their exceptional heating behavior can be even further enhanced by cluster formation. For further studies, we propose a systematic investigation of the relations between the cluster size and MHT performance for embedded nanoflowers, ideally accompanied by polarized SANS studies to probe the interparticle moment correlations.

We want to thank the Institut Laue-Langevin for provision of beamtime at the instrument D33 and David González-Alonso for his help during the experiment. This project received funding from the European Commission Framework Programme 7 under Grant Agreement No. 604448 (NanoMag), the National Research Fund of Luxembourg (No. CORE SANS4NCC Grant), and the Spanish Government (No. MAT2017-83631-C3-R).

\section{REFERENCES}

${ }^{1}$ Q. Pankhurst, N. Thanh, S. Jones, and J. Dobson, "Progress in applications of magnetic nanoparticles in biomedicine,” J. Phys. D 42, 224001 (2009).

${ }^{2}$ P. Southern and Q. A. Pankhurst, "Commentary on the clinical and preclinical dosage limits of interstitially administered magnetic fluids for therapeutic hyperthermia based on current practice and efficacy models," Int. J. Hyperthermia 34, 671-686 (2018).

${ }^{3}$ E. Zhang, M. F. Kircher, M. Koch, L. Eliasson, S. N. Goldberg, and E. Renström, "Dynamic magnetic fields remote-control apoptosis via nanoparticle rotation," ACS Nano 8, 3192-3201 (2014). 
${ }^{4}$ A. M. Master, P. N. Williams, N. Pothayee, N. Pothayee, R. Zhang, H. M. Vishwasrao, Y. I. Golovin, J. S. Riffle, M. Sokolsky, and A. V. Kabanov, "Remote actuation of magnetic nanoparticles for cancer cell selective treatment through cytoskeletal disruption," Sci. Rep. 6, 33560 (2016).

${ }^{5}$ D. Eberbeck, F. Wiekhorst, U. Steinhoff, and L. Trahms, "Aggregation behaviour of magnetic nanoparticle suspensions investigated by magnetorelaxometry," J. Phys.: Condens. Matter 18, S2829 (2006).

${ }^{6}$ L. Gutierrez, M. Moros, E. Mazario, S. de Bernardo, J. M. de la Fuente, M. del Puerto Morales, and G. Salas, "Aggregation effects on the magnetic properties of iron oxide colloids," Nanotechnology 30, 112001 (2019).

${ }^{7}$ R. Di Corato, A. Espinosa, L. Lartigue, M. Tharaud, S. Chat, T. Pellegrino, C. Ménager, F. Gazeau, and C. Wilhelm, "Magnetic hyperthermia efficiency in the cellular environment for different nanoparticle designs," Biomaterials 35, 6400-6411 (2014)

${ }^{8}$ E. A. Périgo, G. Hemery, O. Sandre, D. Ortega, E. Garaio, F. Plazaola, and F. J. Teran, "Fundamentals and advances in magnetic hyperthermia," Appl. Phys. Rev. 2, 041302 (2015).

${ }^{9}$ B. Sanz, M. P. Calatayud, E. De Biasi, E. Lima, Jr., M. V. Mansilla, R. D. Zysler, M. R. Ibarra, and G. F. Goya, "In silico before in vivo: How to predict the heating efficiency of magnetic nanoparticles within the intracellular space," Sci. Rep. 6, 38733 (2016).

${ }^{10}$ J.-L. Déjardin, F. Vernay, M. Respaud, and H. Kachkachi, "Effect of dipolar interactions and dc magnetic field on the specific absorption rate of an array of magnetic nanoparticles," J. Appl. Phys. 121, 203903 (2017).

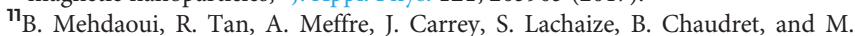
Respaud, "Increase of magnetic hyperthermia efficiency due to dipolar interactions in low-anisotropy magnetic nanoparticles: Theoretical and experimental results," Phys. Rev. B 87, 174419 (2013).

${ }^{12}$ M. Sadat, R. Patel, J. Sookoor, S. L. Bud'ko, R. C. Ewing, J. Zhang, H. Xu, Y. Wang, G. M. Pauletti, D. B. Mast, and D. Shi, "Effect of spatial confinement on magnetic hyperthermia via dipolar interactions in $\mathrm{Fe}_{3} \mathrm{O}_{4}$ nanoparticles for biomedical applications," Mater. Sci. Eng., C 42, 52-63 (2014).

${ }^{13}$ C. Blanco-Andujar, D. Ortega, P. Southern, Q. Pankhurst, and N. Thanh, "High performance multi-core iron oxide nanoparticles for magnetic hyperthermia: Microwave synthesis, and the role of core-to-core interactions," Nanoscale 7, 1768-1775 (2015).

${ }^{14}$ I. Andreu, E. Natividad, L. Solozabal, and O. Roubeau, "Nano-objects for addressing the control of nanoparticle arrangement and performance in magnetic hyperthermia," ACS Nano 9, 1408-1419 (2015).

${ }^{15}$ D. F. Coral, P. Mendoza Zélis, M. Marciello, M. d P. Morales, A. Craievich, F. H. Sánchez, and M. B. Fernández van Raap, "Effect of nanoclustering and dipolar interactions in heat generation for magnetic hyperthermia," Langmuir 32, 1201-1213 (2016)

${ }^{16}$ L. Lartigue, P. Hugounenq, D. Alloyeau, S. P. Clarke, M. Lévy, J.-C. Bacri, R. Bazzi, D. F. Brougham, C. Wilhelm, and F. Gazeau, "Cooperative organization in iron oxide multi-core nanoparticles potentiates their efficiency as heating mediators and mri contrast agents," ACS Nano 6, 10935-10949 (2012).

${ }^{17} \mathrm{~S}$. Dutz and R. Hergt, "Magnetic particle hyperthermia-A promising tumour therapy?," Nanotechnology 25, 452001 (2014).

${ }^{18}$ A. Kostopoulou, K. Brintakis, M. Vasilakaki, K. Trohidou, A. Douvalis, A. Lascialfari, L. Manna, and A. Lappas, "Assembly-mediated interplay of dipolar interactions and surface spin disorder in colloidal maghemite nanoclusters," Nanoscale 6, 3764-3776 (2014).

${ }^{19}$ D. Sakellari, K. Brintakis, A. Kostopoulou, E. Myrovali, K. Simeonidis, A. Lappas, and M. Angelakeris, "Ferrimagnetic nanocrystal assemblies as versatile magnetic particle hyperthermia mediators," Mater. Sci. Eng., C 58, 187-193 (2016).

${ }^{20}$ H. Gavilán, A. Kowalski, D. Heinke, A. Sugunan, J. Sommertune, M. Varón, L. K. Bogart, O. Posth, L. Zeng, D. González-Alonso, C. Balceris, J. Fock, E. Wetterskog, C. Frandsen, N. Gehrke, C. Grüttner, A. Fornara, F. Ludwig, S. Veintemillas-Verdaguer, C. Johansson, and M. P. Morales, "Colloidal flowershaped iron oxide nanoparticles: Synthesis strategies and coatings," Part. Part. Syst. Charact. 34, 1700094 (2017).

${ }^{21}$ H. Gavilán, E. H. Sánchez, M. E. Brollo, L. Asín, K. K. Moerner, C. Frandsen, F. J. Lázaro, C. J. Serna, S. Veintemillas-Verdaguer, M. P. Morales, and L. Gutiérrez, "Formation mechanism of maghemite nanoflowers synthesized by a polyol-mediated process,” ACS Omega 2, 7172-7184 (2017).
${ }^{22}$ M. Wetegrove, K. Witte, W. Bodnar, D.-E. Pfahl, A. Springer, N. Schell, F. Westphal, and E. Burkel, "Formation of maghemite nanostructures in polyol: Tuning the particle size via the precursor stoichiometry," CrystEngComm 21, 1956-1966 (2019).

${ }^{23}$ S. Shaw, A. Biswas, A. Gangwar, P. Maiti, C. Prajapat, S. S. Meena, and N. Prasad, "Synthesis of exchange coupled nanoflowers for efficient magnetic hyperthermia," J. Magn. Magn. Mater. 484, 437-444 (2019).

${ }^{24}$ J. Alonso, M. Fdez-Gubieda, J. Barandiarán, A. Svalov, L. F. Barquín, D. A. Venero, and I. Orue, "Crossover from superspin glass to superferromagnet in $\mathrm{Fe}_{\mathrm{x}} \mathrm{Ag}_{100-\mathrm{x}}$ nanostructured thin films $(20 \leq x \leq 50)$," Phys. Rev. B 82, 054406 (2010).

${ }^{25} \mathrm{~S}$. Dutz, "Are magnetic multicore nanoparticles promising candidates for biomedical applications?," IEEE Trans. Magn. 52, 1-3 (2016).

${ }^{26}$ F. Döbrich, M. Elmas, A. Ferdinand, J. Markmann, M. Sharp, H. Eckerlebe, J. Kohlbrecher, R. Birringer, and A. Michels, "Grain-boundary-induced spin disorder in nanocrystalline gadolinium,” J. Phys.: Condens. Matter 21, 156003 (2009).

${ }^{27}$ P. Bender, J. Fock, C. Frandsen, M. F. Hansen, C. Balceris, F. Ludwig, O. Posth, E. Wetterskog, L. K. Bogart, P. Southern, W. Szczerba, L. Zeng, K. Witte, C. Grüttner, F. Westphal, D. Honecker, D. González-Alonso, L. Fernández Barquín, and C. Johansson, "Relating magnetic properties and high hyperthermia performance of iron oxide nanoflowers," J. Phys. Chem. C 122, 3068-3077 (2018).

${ }^{28}$ P. Bender, J. Fock, M. Hansen, L. Bogart, P. Southern, F. Ludwig, F. Wiekhorst, W. Szczerba, L. Zeng, D. Heinke, N. Gehrke, M. T. Fernández Díaz, D. González-Alonso, J. I. Espeso, J. Rodríguez Fernández, and C. Johansson, "Influence of clustering on the magnetic properties and hyperthermia performance of iron oxide nanoparticles," Nanotechnology 29, 425705 (2018).

${ }^{29}$ A. Lak, M. Cassani, B. T. Mai, N. Winckelmans, D. Cabrera, E. Sadrollahi, S. Marras, H. Remmer, S. Fiorito, L. Cremades-Jimeno, F. J. Litterst, F. Ludwig, M. Liberato, F. J. Teran, S. Bals, and T. Pellegrino, "Fe ${ }^{2+}$ deficiencies, FeO subdomains, and structural defects favor magnetic hyperthermia performance of iron oxide nanocubes into intracellular environment," Nano Lett. 18, 6856-6866 (2018).

${ }^{30}$ A. A. Kuznetsov, "Zero-field and field-induced interactions between multicore magnetic nanoparticles,” Nanomaterials 9, 718 (2019).

${ }^{31}$ P. Bender, E. Wetterskog, D. Honecker, J. Fock, C. Frandsen, C. Moerland, L. K. Bogart, O. Posth, W. Szczerba, H. Gavilán, R. Costo, M. T. Fernández-Díaz, D. González-Alonso, L. Fernández Barquín, and C. Johansson, "Dipolar-coupled moment correlations in clusters of magnetic nanoparticles," Phys. Rev. B 98, 224420 (2018).

${ }^{32}$ P. Bender, L. Fernández Barquín, M.-L. Fdez-Gubieda, D. González-Alonso, D. Honecker, L. Marcano, and W. Szczerba, Spin Correlation in Clusters of Magnetic Nanoparticles (Institut Laue-Langevin (ILL), 2017).

${ }^{33}$ C. D. Dewhurst, I. Grillo, D. Honecker, M. Bonnaud, M. Jacques, C. Amrouni, A. Perillo-Marcone, G. Manzin, and R. Cubitt, "The small-angle neutron scattering instrument D33 at the Institut Laue-Langevin,” J. Appl. Crystallogr. 49, 1-14 (2016).

${ }^{34}$ K. L. Krycka, R. A. Booth, C. R. Hogg, Y. Ijiri, J. A. Borchers, W. Chen, S. M. Watson, M. Laver, T. R. Gentile, L. R. Dedon, S. Harris, J. J. Rhyne, and S. A. Majetich, "Core-shell magnetic morphology of structurally uniform magnetite nanoparticles," Phys. Rev. Lett. 104, 207203 (2010).

${ }^{35}$ I. Orue, L. Marcano, P. Bender, A. García-Prieto, S. Valencia, M. A. Mawass, D. Gil-Cartón, D. Alba Venero, D. Honecker, A. García-Arribas, L. Fernández Barquín, A. Muela, and M. L. Fdez-Gubieda, "Configuration of the magnetosome chain: A natural magnetic nanoarchitecture," Nanoscale 10, 7407-7419 (2018).

${ }^{36}$ S. Mühlbauer, D. Honecker, É. A. Périgo, F. Bergner, S. Disch, A. Heinemann, S. Erokhin, D. Berkov, C. Leighton, M. R. Eskildsen, and A. Michels, "Magnetic small-angle neutron scattering," Rev. Mod. Phys. 91, 015004 (2019).

${ }^{37}$ D. Honecker, A. Ferdinand, F. Döbrich, C. D. Dewhurst, A. Wiedenmann, C. Gómez-Polo, K. Suzuki, and A. Michels, "Longitudinal polarization analysis in small-angle neutron scattering," Eur. Phys. J. B 76, 209-213 (2010).

${ }^{38}$ J. S. Pedersen, "Analysis of small-angle scattering data from colloids and polymer solutions: Modeling and least-squares fitting," Adv. Colloid Interface Sci. 70, 171-210 (1997).

${ }^{39} \mathrm{~T}$. Li, A. J. Senesi, and B. Lee, "Small angle x-ray scattering for nanoparticle research,” Chem. Rev. 116, 11128-11180 (2016). 
${ }^{40}$ D. Alba Venero, S. Rogers, S. Langridge, J. Alonso, M. Fdez-Gubieda, A. Svalov, and L. Fernández Barquín, "Magnetic nanoscopic correlations in the crossover between a superspin glass and a superferromagnet," J. Appl. Phys. 119, 143902 (2016).

${ }^{41}$ D. Honecker, L. F. Barquín, and P. Bender, "The magnetic structure factor of correlated nanoparticle moments in small-angle neutron scattering," preprint arXiv:1904.06243 (2019).

${ }^{42}$ P. Bender, L. Bogart, O. Posth, W. Szczerba, S. Rogers, A. Castro, L. Nilsson, L. Zeng, A. Sugunan, J. Sommertune, A. Fornara, D. González-Alonso, L. Fernández Barquín, and C. Johansson, "Structural and magnetic properties of multi-core nanoparticles analysed using a generalised numerical inversion method," Sci. Rep. 7, 45990 (2017).

${ }^{43}$ A. Urtizberea, E. Natividad, A. Arizaga, M. Castro, and A. Mediano, "Specific absorption rates and magnetic properties of ferrofluids with interaction effects at low concentrations," J. Phys. Chem. C 114, 4916-4922 (2010).

${ }^{44}$ D. Serantes, D. Baldomir, C. Martinez-Boubeta, K. Simeonidis, M. Angelakeris, E. Natividad, M. Castro, A. Mediano, D.-X. Chen, A. Sanchez, L. Balcells, and B. Martínez, "Influence of dipolar interactions on hyperthermia properties of ferromagnetic particles," J. Appl. Phys. 108, 073918 (2010). 